\section{Cahiers de Narratologie}

Analyse et théorie narratives

$28 \mid 2015$

Le récit comme acte cognitif

\title{
Cognition et Hybridité. Le lecteur dans la zone grise
}

\section{Arnaud Schmitt}

\section{(2) OpenEdition \\ Journals}

Electronic version

URL: https://journals.openedition.org/narratologie/7201

DOI: 10.4000/narratologie. 7201

ISSN: 1765-307X

Publisher

LIRCES

\section{Electronic reference}

Arnaud Schmitt, "Cognition et Hybridité. Le lecteur dans la zone grise", Cahiers de Narratologie [Online], 28 | 2015, Online since 29 October 2015, connection on 11 June 2021. URL: http://

journals.openedition.org/narratologie/7201; DOI: https://doi.org/10.4000/narratologie.7201

This text was automatically generated on 11 June 2021.

Article L.111-1 du Code de la propriété intellectuelle. 


\title{
Cognition et Hybridité. Le lecteur dans la zone grise
}

\author{
Arnaud Schmitt
}

1 Il ne s'agit pas ici de rouvrir le débat sur l'identité générique de l'autofiction, ni d'en rappeler son potentiel et ses impasses intrinsèques ${ }^{1}$. Nous allons poser la question de la réception de ces textes - je parle plus largement ici de ces autobiographies équivoques ou encore des écrits historiques un peu trop omniscients ou romancés-, et de l'expérience de lecture qu'ils suscitent; en d'autres termes, que signifie réellement pour le lecteur " osciller et vaciller ${ }^{2}$ » face à ces textes, en partant du postulat qu'il est tout à fait possible, d'un point de vue cognitif, que cela puisse ne rien signifier.

2 Pour décrire rapidement et précisément l'avancée du débat sur l'hybridité générique, il est raisonnable d'affirmer que le panfictionalisme semble avoir pour l'instant remporté la partie. Celui-ci nous invite donc à nous accommoder du fait, contre-intuitif pour certains (dont, autant le dire dès à présent pour clarifier les choses, je fais partie), que lorsqu'il s'agit de récit, la ségrégation générique entre écriture factuelle et écriture fictionnelle n'a plus de raison d'être. Nous sommes à l'opposé de l'une des pierres de touche de l'approche rationaliste, appelée aussi "ségrégationniste ", à savoir le pacte autobiographique conceptualisé par Philippe Lejeune, pas uniquement dans sa version originelle (1975), mais aussi dans celle plus récente de $2005^{3}$ qui s'articule toujours autour "du "pacte de vérité" qui donne leur force à ces signes de vies » (9) : «Je suis toujours l'auteur du Pacte autobiographique, qui croit, avec Rousseau, à l'engagement de vérité. Mais aussi l'auteur des Brouillons de soi, qui sait la mobilité de la vie » (9). Trente ans après la publication de son texte séminal, Lejeune persiste et signe en affirmant que le «pacte autobiographique est l'engagement que prend un auteur de raconter directement sa vie (ou une partie, ou un aspect de sa vie) dans un esprit de vérité » (31). La «mobilité de la vie» n'est donc pas incompatible selon lui avec une forme d'engagement vis-à-vis du lecteur.

3 Mais à l'autre bout du spectre critique, les notions de vérité, de sincérité, voire d'engagement, n'ont que peu de sens. Le panfictionalisme se présente sous diverses formes. Pour n'en nommer que quelques-unes : 
- tout d'abord ce que nous pourrions appeler l'école française de l'autofiction qui revendique l'indétermination du texte, le droit de ce dernier et de son auteur à ne pas répondre aux questions indiscrètes ou tout simplement inutiles du type « de quel genre s'agit-il ? » ou encore «est-ce vraiment ainsi que cela s'est passé ?» (Doubrovksy, Colonna, Vilain...).

- ensuite, la littérature postmoderne qui, à l'instar d'un Philip Roth ou d'un John Fowles, s'est adonnée à la métalepse de l'auteur par exemple ou, tout simplement, au brouillage des identités et des cartes génériques, Linda Hutcheon faisant très justement remarquer dans son ouvrage de référence sur la question postmoderne que la frontière la plus souvent mise à mal par les romanciers postmodernes fut celle séparant l'art et la vie $^{4}$.

- enfin, en dernier lieu, tout simplement les théoriciens de la littérature qui se sont intéressés à ces textes qui offrent prétendument un double pacte de lecture, tel Philippe Gasparini qui a écrit à travers son diptyque ${ }^{5}$ la version française de l'étude de cette zone grise à laquelle les américains se réfèrent par le biais de ce qui devrait normalement demeurer un oxymore : romans autobiographiques. Mais Gasparini n'est pas le seul à promouvoir le concept d'hybridité comme solution au problème posé par l'autofiction ${ }^{6}$, les spécialistes étasuniens de la question en font de même. Pour Paul John Eakin dont les écrits sur la question font référence, «la fiction et le processus de création fictionnelle sont un élément central de n'importe quelle vie telle qu'elle est vécue et de n'importe quelle œuvre artistique consacrée à la présentation de cette $v_{i e}{ }^{7} »$. Pour Timothy Dow Adams, les arguments qui opposent fiction et écrits référentiels ne sont plus vraiment recevables depuis l'avènement du nouveau journalisme (ce que nos collègues outre-Atlantique appellent aussi faction) et de ses diverses variations postmodernes ${ }^{8}$. Enfin, la version la plus récente mais aussi la plus absolue du panfictionalisme - car il faut bien admettre que cette approche est aussi une affaire de degrés chez les penseurs que je viens de nommer - est incarnée par David Shields et plus précisément par la radicalité théorique développée dans son diptyque publié récemment". Selon lui, la question même de l'hybridité est obsolète; et quant à se poser la question de la véracité d'un texte, cela dépasse l'entendement. Voici un florilège des raisons avancées : "une part incroyablement grande de la fiction est immensément autobiographique et beaucoup d'écrits référentiels sont hautement imaginés ${ }^{10}$ » ; « la ligne qui sépare fait et fiction est plus vague que ce que la plupart de gens veulent bien avouer ${ }^{11}$ »; " une partie ce qui se fait de mieux dans la fiction est maintenant écrite en tant que non-fiction ${ }^{12} »$. Cependant certains chercheurs travaillant sur des champs similaires, et arrivant parfois à des conclusions analogues, restent néanmoins conscients des conséquences d'une telle éradication des frontières, notamment sur le plan éthique. C'est le cas de Leigh Gilmore qui se méfie de l'éternel argument selon lequel la fiction permet d'avoir accès à des vérités interdites aux faits : "Une fois que la vérité de la fiction est préférée à celle des faits, l'autorité à la fois du traumatisme et de l'autobiographie qui dérive de la crédibilité du témoin est remise en cause et risque de perdre sa légitimité ${ }^{13}$ ".

5 Je n'ai pas l'intention d'aborder plus longuement ici cette crise de « légitimité », même si les adeptes du panfictionalisme l'ont à mon avis un peu trop rapidement balayée d'un revers de la main. Par contre, au-delà de son aspect éthique, le panfictionalisme pose de sérieux problèmes phénoménologiques, herméneutiques et tout simplement cognitifs qui, il me semble, restent encore à explorer, malgré la révolution paradigmatique proposée par cette approche radicale. En effet ceux qui adhèrent à la thèse de ce que 
nous pourrions donc appeler une hybridité indéterminée se rangent souvent derrière la bannière du panfictionalisme, plus par renoncement que par conviction ; renoncement à démêler le fameux écheveau autofictionnel ou postmoderne. Renoncer ne résout toutefois pas le problème. De plus, cette hybridité a-t-elle réellement été définie ? D'un point de vue neutre, essentialiste, oui, probablement. Mais du point de vue de la réception, d'une expérience de lecture, peut-on dire ce que l'hybridité signifie, comment elle est vécue ? C'est donc ce que je me propose de faire ici, par le truchement de la poétique cognitive.

Deux remarques préalables méritent d'être faites :

7 1) Comme Philippe Gasparini l'a démontré dans son ouvrage consacré à l'autofiction, l'hybridité implique un double pacte qui se manifeste par des oscillations ou des vacillements dans la réception, pour reprendre les termes de Susan S. Lanser. De plus, Gasparini, contrairement à d'autres, a bien insisté sur le rôle prépondérant du paratexte dans cette indécision. Le résultat est donc un «Je » double, un «Je » qui est " déictiquement double». Je reviendrai sur cette notion mais il me semble important de se concentrer d'ores et déjà sur ce double "Je » qui, dans la vie réelle, n'existe pas et relève de la narratologie non naturelle (comme la plupart des mondes possibles inhérents à la science-fiction par exemple). D'un point de vue déictique, «Je » est celui qui est là, qui parle à partir d'une réalité déictique (et j'associe ici la notion de présence à celle d'identité, de présence identitaire). Et même celui qui ment demeure déictiquement univoque, la base énonciative - notion essentielle sur laquelle je conclurai cet article - reste claire. De plus, l'hybridité impliquerait une extrême flexibilité herméneutique qui dans les actes de lecture quotidiens reste rare. Lethcoe parle de la " règle de continuité » selon laquelle nous - et j'emploie le «nous » à contrecœur pour des raisons qui vont vite s'avérer évidentes - choisissons majoritairement « le chemin de la moindre résistance ${ }^{14}$ » qui nous permet de garder notre cadre (frame) de référence premier (autobiographie ou fiction par exemple, position de générique "pré-lecture " souvent basée sur l'appréhension du paratexte par exemple). Cette règle est assez proche de celle énoncée par Monika Fludernik, baptisée « règle de préférence » : « une fois que le lecteur a établi une perspective qui prévaut, il tente de la conserver aussi longtemps que possible ${ }^{15} »$. D'un point de vue cognitif, cela signifie que nous ne changeons de cadre qu'en dernier recours, confrontés à une forme de désorientation ou de perte sémantique. Un lecteur a donc généralement tendance à éviter les surcharges cognitives ; ou, pour le dire de manière plus crue, certains lecteurs sont « fainéants ». Il s'agit bien sûr d'une simplification mais, cognitivement, nous sommes tous plus ou moins «fainéants ", et c'est une bonne chose. Nous passons notre vie à simplifier notre perception du réel. Dans son remarquable ouvrage, How Literature Plays with the Brain: the Neuroscience of Reading and Art, Paul B. Armstrong évoque justement cette propension du cerveau à rendre routinières certaines opérations ${ }^{16}$. Tout ce qui est nouveau est par exemple uniquement appréhendé par le biais de ce qui est déjà établi catégoriquement, par les cadres du passé17. Nous pensons par TC (types cognitifs) et schémas narratifs également nommés « séquençage des événements ». Il s'agit aussi de s'économiser car, comme le faisait remarquer Umberto Eco, «tout texte est une machine paresseuse qui réclame une active coopération interprétative de la part de son destinataire " (283), une machine à trous que le lecteur doit combler, à mondes possibles que le lecteur doit imaginer, sachant aussi que " plus la définition sera basse et l'objet inconnu [et cela vaut pour la mimèsis autant que pour le genre du texte], plus le processus inférentiel requis sera important» (388). Cette logique de cadre et de 
résistance évoque une dialectique familière aux sciences cognitives, l'effet de primauté et l'effet de récence (primacy effect / recency effect) présentée ainsi par Manfred Jahn : "La primauté et la récence sont des mécanismes cognitifs qui peuvent être expliqués de manière utile dans le contexte des notions de cadres et de préférence. Normalement, on peut imaginer qu'un cadre a deux instincts quasi-organiques: il essaie de se protéger, et d'élargir au maximum sa portée ${ }^{18} »$. Notre vie cognitive est ainsi déchirée entre deux tendances majeures: a) la règle de préférence de primauté (primacy preference rule), qui nous pousse à garder le même cadre aussi longtemps que possible ; b) la règle de préférence de récence (recency preference rule), qui permet de remplacer un cadre lorsque cela est nécessaire. En matière d'interprétation littéraire, certains textes encouragent plutôt les lecteurs à conserver leurs cadres, d'autres à les renouveler. Cependant, « la mise en place des structures neuronales est longue et il est peu probable qu'une seule lecture d'un texte en particulier les transforme ${ }^{19}$ ». Comme cela a été souligné par Fludernik et Lethcoe, nous tentons dans la mesure du possible de stabiliser nos cadres, et parfois même lorsque des informations nous inciteraient à procéder autrement. Armstrong montre que d'un point de vue neurobiologique, le mode opératoire est similaire : "Confronté à la nouveauté ou à une anomalie, celui ou celle qui interprète n'efface pas ses schémas de réception habituels pour recommencer à zéro mais, au lieu de cela, révise et étend le familier afin de s'adapter à l'inconnu ${ }^{20}$ ". Il ajoute que le cerveau a beau être flexible et adaptable, "cette plasticité est confrontée à des limites " ("this plasticity is constrained », 32). Bien que différente d'un lecteur à un autre, cette propension cognitive conservatrice a un rapport direct avec la notion d'hybridité. En effet, les théoriciens de la fameuse zone grise (à la fois factuelle et fictionnelle) ont trop souvent fait l'économie d'une définition précise de l'hybridité à laquelle ils se référaient. Je vois trois types possibles d'hybridité : 1) deux cadres qui se superposent ;2) deux cadres distincts qui alternent ; 3) un cadre unique, et donc nouveau, formé à partir des deux anciens cadres. Dans le dernier cas, nous ne sommes plus dans un contexte hybride, mais dans celui d'un nouveau genre qui a su émerger à partir d'un processus de transversalité générique qui s'est inscrit dans une certaine durée historique. Rien de vraiment hybride ici, il s'agit simplement d'une mutation générique. On pourrait ainsi avancer que l'autofiction est le produit de la rencontre entre le récit autobiographique et la métalepse de l'auteur dans sa version postmoderne ; un cadre unique est suffisant dans ce cas précis. La deuxième éventualité n'implique pas non plus une authentique hybridité puisque deux cadres se substituent l'un à l'autre, successivement; pour traduire cela de manière empirique, cela reviendrait à changer constamment d'avis quant à la nature du texte pendant l'acte de lecture. La seule hybridité qui me semble recevable est celle décrite dans le premier cas de figure, mais est-elle réaliste pour autant? Peut-on réellement superposer deux cadres pour percevoir un seul objet? Ou percevoir simultanément deux objets différents? Il n'y a peut-être pas encore de réponse définitive à cette question qui demeure selon moi la question centrale posée par cette zone grise, mais la neurobiologie commence à nous donner des indices de réponse. Les expériences menées sur ce qu'on appelle "multi-stable images», c'est-à-dire des images représentant, par exemple, à la fois une tête de lapin et de canard pour reprendre le célèbre exemple de Gombrich, tendent à démontrer que nous pouvons alterner entre ces figures, mais sans pouvoir les faire cohabiter : «En termes neurologiques, chacune de ces trois configurations (canard, lapin ou canard-lapin) entraînerait une synthèse corticale quelque peu différente, créant une association différente de neurones, 
chacune maintenant temporairement les autres à distance ${ }^{21}[. .]$.$» . Dans tous les cas, la$ réponse apportée par le panfictionalisme apparaît trop désinvolte. Mais une chose est certaine quelle que soit l'approche privilégiée : une absence totale de cadre n'est pas de l'ordre du possible.

8 2) La seconde remarque préalable que je souhaiterais faire vient quelque peu relativiser la première. J'ai précisé que le pronom personnel «nous » était particulièrement trompeur lorsqu'on tentait de désigner une expérience de lecture. Ainsi, cette recherche de "la moindre résistance " n'est pas commune à tous les lecteurs. La théorie de l'hybridité a pourtant justement postulé le contraire : les lecteurs sont prêts à modifier leurs cadres sans réticence particulière, ce qui peut sembler peu réaliste. Le problème réside dans le fait que, bien souvent, le théoricien, généralement universitaire, a, à l'instar du romancier, un lecteur idéal en tête et que, dans la plupart des cas, ce lecteur idéal occupe le bureau adjacent au sien. Or, la lecture " professionnelle » qui est la nôtre est un mode de lecture altéré, en plusieurs temps, qui ne correspond pas forcément à une lecture unique, expérience plus commune. D'ailleurs, je suis persuadé qu'une majorité d'entre nous, lorsque nous lisons un texte sur lequel nous ne serons pas amenés à travailler directement, obéit à une logique d'immersion et de représentation pas forcément différente de celle d'un lecteur type (même si du fait de la multitude des affects et des percepts générée par une œuvre de fiction, un tel lecteur n'existe pas). Néanmoins la «capacité négative» («negative capability 》) appelée de ses vœux par Keats, cette " capacité à contempler le monde sans le désir de tenter de réconcilier ses aspects contradictoires ou de le faire rentrer dans des systèmes rationnels et clos", est finalement ce qui se rapproche le plus de la première version de l'hybridité mentionnée plus haut. Je ne sais pas si certaines études cognitives mesurent le degré de sophistication d'un lecteur, mais nous pouvons nous référer à l'ouvrage récent de Daniel Kahneman, expert international en psychologie cognitive et prix Nobel d'économie, Thinking, Fast and Slow ${ }^{22}$. Dans cet ouvrage, Kahneman explore justement le thème de la paresse cognitive, ou du moins de l'économie de moyens cognitifs, et souligne les efforts dont nous sommes heureusement occasionnellement capables lorsque la situation l'exige. Il divise notre travail cognitif en deux systèmes, système 1 et système 2 en précisant que nous nous en remettons autant que possible au premier système car «il opère automatiquement et rapidement, avec pas ou peu d'effort et aucune impression de contrôle émanant de notre volonté » (20) alors que le système 2 "répartit l'attention nécessaire vers les activités mentales qui réclament un effort ", plus particulièrement pour les opérations que l'on associe aux "expériences subjectives relatives au libre choix et à la concentration» (21). Il est donc vrai que, normalement, nous «évitons la surcharge cognitive en divisant nos tâches en de multiples étapes simples » et obéissons à « la loi du moindre effort» (38). On revient à cette réticence au changement, et plus particulièrement au changement de cadre, théorisée par Fludernik ou Lethcoe; réticence qui ne laisse que peu de place à l'appréhension d'une combinaison aussi sophistiquée que l'hybridité générique. Kahneman ouvre néanmoins la porte à un système 2 qui serait alors capable d'appréhender la capacité négative nécessaire à l'existence de la zone grise qui nous intéresse ici. En effet, bien que minoritaire, le système 2 peut gérer le doute, tout simplement parce qu'il peut envisager simultanément « des possibilités incompatibles» (114). Mais Kahneman n'omet pas de préciser que nos systèmes 2 sont inégaux et peuvent accomplir des tâches plus ou moins élaborées. En partant du préalable que l'hybridité est une alternance indécise, 
voire indémêlable, certains systèmes 2 pourraient donc soutenir cette indétermination. Nous devons donc, comme Daniel Kahneman nous exhorte à le faire, "accepter les complexités d'une perspective hybride » (402). Je tenais à faire ce détour par l'ouvrage du chercheur américano-israélien pour tenter de rentrer un peu plus dans la spécificité de la réception de la zone grise et reconnaître que les attentes de chaque lecteur et les efforts auxquels il ou elle consent varient énormément. En d'autres termes, «la façon dont le cerveau peut effectuer des nouvelles expériences dépend du répertoire qui est déjà à sa disposition ${ }^{23}$ ", et nous avons tous des répertoires différents.

Ces observations préalables faites, nous allons maintenant nous tourner vers les deux problèmes se trouvant au cœur de notre problématique, à savoir la double nature de l'hybridité et le centre déictique d'où part l'énonciation.

10 Malgré la tentative hautement respectable de Dorrit Cohn dans La Distinction de la Fiction ${ }^{24}$ d'accorder à l'écriture fictionnelle une irréfutable spécificité, je reste persuadé, suivant le célèbre adage de Searle, que la distinction entre la fiction, l'autobiographie et l'autofiction opérée à partir d'une base essentialiste est vouée à l'échec car la fiction est polymorphe et trouvera toujours un moyen de singer ce dont elle souhaite s'inspirer. Jean-Marie Schaeffer a d'ailleurs en quelque sorte clos le débat en démontrant dans Pourquoi la fiction? que «l'idée selon laquelle il y aurait une modalité de représentation qui serait spécifiquement fictionnelle et qui se distinguerait d'une modalité factuelle ne me semble pas correcte »; en effet, il conclut ce débat ouvert il y a bien longtemps par une phrase qui me semble faire autorité de par son bon sens, mais aussi grâce aux arguments avancés dans son ouvrage: «Il n'existe qu'une seule modalité représentationnelle» (109). De plus, et bien que cette notion soit essentielle pour déterminer notre horizon d'attente, qui n'est rien d'autre qu'un cadre cognitif, le genre textuel est une valeur immatérielle : il n'existe qu'en tant que position de réception, dans l'esprit du lecteur. Aucune empreinte générique inhérente à un ouvrage (paratexte, hypertexte...) n'est fiable puisqu'elle peut être détournée, incomprise, mal comprise, ou encore feinte. Cette indétermination a un corollaire hautement problématique : il n'y a formellement aucune différence entre un réel imaginé et un réel dépeint. Mais, qu'en est-il d'un référent réel apparaissant dans un texte fictionnel ? Pour répondre à cette question, il faut revenir à l'origine même de l'énigme posée par l'hybridité: la différence entre un référent fictionnel et factuel.

11 Dans Literature and the Brain ${ }^{25}$, Norman Holland nous explique que notre perception du réel - qui consiste finalement plus en une reconstruction à l'aide de ressources cérébrales multiples à la suite d'une perception, qui nous ramène au binding problem théorisé entre autres par Antonio Damasio - s'organise grossièrement autour de deux systèmes, le système " où » et le système "quoi » (the "where" system and the "what" system), le second système étant le plus élaboré, même si ces deux systèmes ont pour finalité de produire une perception unifiée. Cependant lorsque nous lisons de la fiction, cette dualité perceptive disparaît et nous avons affaire "à une forme spéciale de reconnaissance d'objet dans laquelle les objets sont des lettres, des graphèmes, des marges, des mots [...] identifiés comme se trouvant dans une certaine région de l'espace ${ }^{26} »$. En d'autres termes, le réel tel qu'il est décrit dans une œuvre de fiction est dépourvu d'incarnation spatiale, de « où ». Un roman peut produire une « modélisation mimétique ${ }^{27}$ » de la réalité, un " quoi » en quelque sorte, mais tout ce qu'il représente se produit dans un «où qui n'a jamais existé». Bien sûr, et cela complique substantiellement le problème, certains lieux évoqués n'ont beau être que des mots, ils 
font cependant référence à des lieux qui ont bel et bien une présence physique dans le monde réel. C'est d'ailleurs ce qui sépare l'autobiographie du roman, par exemple : le «où " de papier a un équivalent physique dans un cas, pas dans l'autre. Lire une autobiographie consiste à recréer un lieu et des événements, pour reprendre la dualité mise à jour par Holland, qui ont existé. De son côté, le lecteur de la fiction est libre d'imaginer son univers spatial de la façon dont il le souhaite. Marie-Laure Ryan établit une distinction fondamentale entre AW (actual world, monde réel) et TAW (textual actual world, monde réel textuel ${ }^{28}$ ), la référence du second est bien le premier, mais son « être de papier " rend cette référence problématique comme on le sait. Pourtant Ryan postule cette équivalence dans le cas, par exemple, de récits historiques : «TAW = AW » (28). La transformation du signe égal en une réalité cognitive n'est toutefois pas aussi simple que cela n'y paraît. De plus, pour revenir au problème évoqué plus haut et qui constitue selon moi le cœur de la confusion générée par l'hybridité, que se passe-t-il lorsque dans un monde fictionnel, un lieu réel est évoqué ? Cette éventualité entre dans ce que Ryan appelle les "relations d'accessibilité » (" accessibility relations») et plus particulièrement les « relations transunivers » («transuniverse relations», 32-33). Elle en donne l'exemple suivant : « la phrase tirée de Guerre et Paix “Le 29 mai [1812], Napoléon quitta Dresde" est vérifiée dans AW, mais son monde de référence est le monde de Guerre et Pai ${ }^{29}$ "; événement similaire, mondes de référence différents donc. Mais la question séminale, à la suite de celles posées plus haut, me semble être la suivante : s'agit-il réellement du même événement ? S'agit-il de la même expérience de lecture (et je me place ici du point de vue cognitif) selon que Napoléon apparaisse dans un roman ou dans un livre d'histoire?

Ces questions sont complexes et nécessiteraient sans aucun doute un dispositif scientifique afin d'accéder le plus directement possible à des expériences de lecture, mais nous pouvons à notre manière commencer à y répondre et proposer des pistes de recherche. Pour Dow Adams, « il est préférable de penser l'autobiographie comme une chose fabriquée à partir d'une chose faite ${ }^{30}$ ». Une "chose faite ", c'est-à-dire un fait, quelque chose qui est extrait du réel, ne peut exister textuellement que si elle est rematérialisée par la fabrique de notre mémoire, zone dangereusement proche de celle occupée par notre imaginaire. Wilfrid Sellars a démontré dans Empiricism and the Philosophy of Mind que le réel en tant que perception reçue n'a rien d'inné ; c'est ce qu'il a appelé le «mythe du donnési ${ }^{31}$. Notre architecture cérébrale est responsable de cet éclatement perceptif et « ce que nous voyons est déterminé autant par l'organisation et les lois qui régissent notre cerveau que par la réalité physique du monde externe ${ }^{32} »$. Armstrong nous rappelle que quelque chose d'aussi prétendument immédiat que la vision est «fondamentalement herméneutique » ("fundamentally hermeneutic »,62) car avant d'être perception, elle est sélection et combinaison. Notre être au monde est intrinsèquement différé : il y a une « rupture temporelle » (« temporal split ») à la base de l'existence humaine car il n'y a pas de simultanéité entre notre perception du réel et l'image que nous en renvoie notre cerveau (Armstrong, 101). Selon Antonio Damasio, " notre conscience est probablement en retard d'environ cinq cents millisecondes ${ }^{33}$ ". Quant à notre économie cognitive, elle se charge de réduire au minimum les données que nous assimilons et cela a un impact direct sur des notions aussi fondamentalement littéraires que la mimésis ou la modélisation mimétique: «Les écrivains réduisent lorsqu'ils écrivent, et les lecteurs réduisent lorsqu'ils lisent. Le cerveau lui-même est construit pour réduire [...]. Le réalisme en littérature est non seulement une fausse idole, mais aussi un but inatteignable ${ }^{34}$ ». La notion d'un réel auquel on accéderait 
directement ou de manière innée s'éloigne donc encore un peu plus lorsque celui-ci doit être retranscrit dans un texte. Cette condition originelle oxymorique, à la fois fait et chose fabriquée, nous renvoie à la nature profondément imparfaite de toute entreprise autobiographique et biographique. De nombreux ouvrages s'appuyant sur des travaux scientifiques ont notamment mis en lumière le rôle à la fois essentiel et pourtant profondément perturbateur joué par la mémoire dans ces entreprises. À la fois peu fiable et pourtant incontournable, la mémoire (collective ou personnelle) est pourtant la ressource principale lorsqu'il s'agit de faire exister le réel à nouveau. Nous nous plaçons ici du point de vue de l'auteur, mais la difficulté n'est pas moindre lorsqu'il s'agit du lecteur. Celui-ci doit donc se représenter du vécu à partir de signes textuels, de conventions rhétoriques qui ne diffèrent pas de celles que l'on peut trouver dans certains romans. De fait, la sensation, toute relative certes, créée en 1979 par l'article de Paul de Man "Autobiography as De-facement ${ }^{35}$ ", semble difficile à comprendre aujourd'hui. Selon de Man, le fait qu'un abîme sépare je narrant et je narré, ce dernier n'étant plus présent (écart qui a notamment été étudié par Jean Starobinski dans La Relation critique $^{36}$ ), devrait nous mener à percevoir l'autobiographie comme une impossibilité, un mode d'écriture reposant essentiellement sur la figure de la prosopopée. Néanmoins cet abîme entre notre moi présent et nos moi passés, et la distance fondamentale suggérée par la prosopopée ne me semblent en rien condamner l'autobiographie à une forme d'obsolescence, bien au contraire. Ce processus reflète de manière très réaliste la situation qui est la nôtre lorsque nous repensons à un événement passé, lorsque nous le racontons à un narrataire : nous ne sommes plus le même et l'événement est révolu, et notre mémoire en est le modérateur auquel nous accordons trop facilement une confiance aveugle. Ce n'est pas l'impossibilité d'une écriture auto-référentielle que de Man pensait décrire, il s'agissait tout simplement de ces « quoi » et de ces « où » qui disparaissent chaque jour. Le délai et la distance sont de toute façon ce qui caractérise notre relation au réel (délai perspectif, comme nous l'avons vu, dans un premier temps, distance narrative dans un second). Et, de plus, Antonio Damasio nous a exhortés à ne pas sous-estimer non plus la grande capacité de notre mémoire - notre " mémoire autobiographique » (" autobiographical memory $\left.{ }^{37} »\right)$ à gérer notre identité (passé, présente et future) à travers le temps.

13 Cependant, cette distance n'est pas l'unique obstacle lorsqu'il faut aborder la représentation textuelle du réel. Dans sa magistrale étude des processus de mimétisme qui sont à la base de la fiction, Jean-Marie Schaeffer nous fait remarquer que tout « enfant doit en effet apprendre à faire la distinction entre ce qui est « pour de vrai » et " ce qui est pour de faux »" (55). Cette distinction qui nous semble essentielle, et sans laquelle les grands types littéraires (et notamment la séparation fiction /vécu) n'existent pas, n'est, elle non plus, pas « donnée » mais acquise. En empruntant d'autres chemins, le cogniticien Richard J. Gerrig ${ }^{38}$ revient sur l'un des plus vieux adages de la théorie littéraire, «the willing suspension of disbelief » de Coleridge (la suspension consentie de l'incrédulité). Gerrig montre en effet que nous avons une propension naturelle à être crédule lorsque nous lisons de la fiction. Pour étayer sa position, Gerrig se réfère à Spinoza selon lequel pour percevoir, nous devons d'abord croire que ce que nous percevons est bien réel. Selon Gerrig, nous appréhendons la fiction de deux manières. Tout d'abord de manière non systématique (unsystematic), nous percevons et ce que nous percevons est perçu comme «vrai ». Et ensuite l'autre système, minoritaire, systématique (systematic), examine réellement la nature de ce que nous percevons (27). Il en vient donc à contester l'adage de Coleridge et à affirmer le contraire : nous croyons 
naturellement en la véracité d'une histoire, l'effort à fournir ne consiste donc pas à suspendre notre incrédulité, mais bien à la construire ("construction of disbelief ", 240). Cette "construction de l'incrédulité " a donc plusieurs corollaires essentiels quand nous lisons un texte ambigu génériquement :

14 la lecture de la fiction n'exige pas de la part du lecteur un effort supplémentaire, bien au contraire.

la nature du texte est encore une fois déterminée par les prédispositions du lecteur visà-vis de ce dernier.

pour que la zone grise devienne une réalité, le lecteur doit adopter une position indéfinie entre crédulité et incrédulité par rapport à ce qu'il lit.

Le dernier corollaire est une question que nous avons déjà posée et à laquelle nous allons tenter de répondre maintenant. Dans le cas où le lecteur ne suspend pas son incrédulité et consomme le texte intégralement comme une œuvre de fiction (qu'elle le soit effectivement ou pas, cela revient au même), comment sont perçues les « relations transunivers ", et dans ce cas précis les personnes réelles et les lieux tels que Napoléon ou Dresde? Dans Postmodernist Fiction, Brian McHale répond en partie à cette question : selon lui, dans le cas d'une métalepse de l'auteur par exemple, figure censée établir " un niveau absolu de réalité » et fournir " une assise ontologique solide », le résultat obtenu est l'inverse de l'effet recherché. La supposée réalité absolue est du coup relativisée, quant à l'assise ontologique, elle est déstabilisée ${ }^{39}$. La réalité que l'acte métafictionnel est censé introduire dans la fiction est en fait dissoute par celui-ci. En dernier lieu, « la supposée réalité absolue de l'auteur devient juste un autre niveau de fiction, et le monde réel s'éloigne encore un peu plus» (197). Autrement dit, la fiction annule la réalité $d u$ réel lorsque un élément référentiel pénètre dans une zone fictionnelle. Cela nous mène à ce que j'appellerais l'illusion de la Rose pourpre du Caire et à la question de savoir si, dans un cas d'hybridité, les deux modes peuvent cohabiter. Woody Allen n'y répond pas de manière tranchée (et je suis bien conscient que ce n'est pas ce que l'on attend de lui); en effet, lorsque l'acteur émerge de l'écran, et ainsi du monde fictionnel, pour rejoindre la réalité et ses couleurs, il modifie le cours du réel et interrompt celui de la fiction. Les deux mondes subissent cette méta-perturbation. La position du cinéaste américain est cependant une position métaphorique d'un auteur qui souhaite illustrer de manière divertissante le cas séculaire de cette membrane semiperméable qui fait que, de temps à autre, lorsque nous baissons notre garde du fait d'un contexte cognitif à faible intensité (comme cela est le cas lorsque nous nous relaxons pour consommer de la fiction populaire), nous laissons le monde fictionnel envahir notre réalité en prenant pour argent comptant ce qui n'est que de la fiction. Pour McHale, le cas de figure présenté dans La Rose pourpre du Caire n'est pas envisageable : lorsqu'un élément traverse l'écran (son exemple de la métalepse de l'auteur illustre le cas inverse, le spectateur, ou plutôt le réalisateur, traverse l'écran pour rejoindre la fiction), il est contaminé par l'univers dans lequel il évolue désormais: pas de cohabitation, mais de la contamination. De la même manière, selon Schaeffer et pour en revenir à l'exemple de Napoléon, le personnage historique perd son historicité une fois immergé dans la fiction: "Les personnes historiques qui interagissent avec les personnages fictifs accomplissent des actions qu'elles n'ont pas pu accomplir "dans la vie réelle", puisqu'elles les mettent en relation avec des personnages inventés. De ce fait, elles se trouvent fictionnalisées ». Cependant, il ajoute que, comme ces personnes conservent leurs noms propres, "elles constituent le point de suture entre univers historique et univers fictif» (140). "Fictionnaliser» équivaut donc à contaminer : 
l'univers dominant soumet l'élément exogène à ses propres règles même si l'effet de "suture" souligné par Jean-Marie Schaeffer reste visible. Telle est d'ailleurs sa fonction la plupart du temps : obéir à une logique disruptive aux conséquences très limitées ; être visible, ni plus ni moins.

Or l'autofiction, ou plus précisément sa théorie, a, selon moi, surestimé la flexibilité de notre travail herméneutique, et sous-estimé le phénomène de contamination exposé cidessus ainsi que l'importance accordée à l'origine de l'énoncé qui permet d'en déterminer la nature, et de jauger l'authenticité de la mémoire épisodique ${ }^{40}$ qui soustend le récit. Relier un énoncé, quelle que soit sa nature, à une source énonciatrice représente l'un des actes cognitifs que nous accomplissons quotidiennement, et ce plusieurs fois, il s'agit de l'identification des sources (source tagging) qui fait partie d'une activité plus large qui est le monitoring des sources (source monitoring). Dans Why We Read Fiction - Theory of Mind and the Novel ${ }^{41}$, Lisa Zunshine montre que cette capacité est aussi pleinement utilisée lorsque nous lisons et plus particulièrement lorsque le texte semble problématique (ce qui va à l'encontre de ce que Keats souhaitait avec sa capacité négative). De plus, elle est aussi mobilisée lorsque nous cherchons à déterminer le genre d'un texte. Zunshine nous rappelle par exemple que «les gens ont toujours apporté beaucoup d'importance à la différence entre les histoires "vraies" et «fausses » et certains furent même prêts à mourir pour le droit d'appeler un mythe un mythe ${ }^{42} »$. Elle ajoute :

Les librairies s'accrochent à la séparation entre "fiction» et, disons, "livre d'histoire » dans leurs politiques de rayonnage. Se peut-il qu'aussi imparfaite soitelle, cette séparation épargne aux clients un effort cognitif considérable consistant à « décider » (inconsciemment, bien sûr), lorsqu'ils commencent la lecture d'un livre, le degré d'identification métareprésentationnelle dont chaque élément de l'histoire aura besoin ${ }^{43}$ ?

17 Les librairies effectuent donc le travail préliminaire pour nous (71). En soi, le source tagging représente la raison même d'exister de la narratologie. Et dans tous les cas, ce besoin d'identifier la source des données qui nous entourent implique que nous avons besoin de situer l'autre d'un point de vue ontologique, mais aussi parfois déictique : or, l'autofiction stipule que cet autre est à deux endroits différents, ou plutôt qu'il est là et n'est pas là (ou pour certains, qu'il est là sans être là). Dans Cognitive Poetics ${ }^{44}$, et plus particulièrement dans le chapitre qu'il consacre à la théorie des déplacements déictiques (Deictic Shift Theory ou DST), Peter Stockwell décrit un roman comme un champ composé de plusieurs zones déictiques («deictic zones», 45-46): la zone "perceptuelle» («perceptual»), celle qui nous intéresse ici et couvre les perceptions des actants de la diégèse, ainsi que la zone «temporelle» («temporal»), « relationnelle» (« relational»), « textuelle» («textual») et enfin « compositionnelle» (« compositional»). Passer d'une zone à une autre est par exemple décrit comme une «poussée » («a push») ou un «saut» («a pop»), des termes qui impliquent des réorientations soudaines. Passer d'un narrateur réel à un narrateur fictionnel est une forme extrême de réorientation déictique puisque cela implique une réorientation générique. L'autofiction souhaite se situer dans ce lieu d'instabilité déictique permanente, mais comme nous l'avons vu, ce genre de surcharge cognitive est rarement soutenu durant la période de concentration prolongée nécessaire à la lecture d'un ouvrage. Ce n'est pas vraiment que la capacité négative soit impossible, elle n'est simplement pas envisageable aussi longtemps que ce que le souhaiterait les tenants d'une hybridité dure (indécision générique totale). Quant à l'option proposée par David 
Shields de ne plus se poser la question, en est-ce réellement une ? Lisa Zunshine nous montre que notre instinct métareprésentationnel est fort, et profondément ancré dans le mode opératoire cognitif de l'être humain; identifier la source d'un énoncé, à plus forte raison lorsque celui-ci se présente sous la forme d'un récit autodiégétique, est un réflexe cognitif rarement suspendu. Certains argueront du fait que l'autofiction ne remet pas en cause l'identité bien réelle du narrateur, mais uniquement la véracité d'une partie des faits narrés. Néanmoins un texte perçu comme fictif signale une intention esthétique spécifique, qui à son tour stipule une position auctoriale particulière, qui va déterminer ensuite la perception du lecteur de la fonction-auteur adéquate ; et celle-ci est une part importante de l'identité de l'auteur que le lecteur se construit. Enfin, se poser la question du vrai et du faux d'un énoncé est une compétence durement acquise au cours de la petite enfance, nous ne la mettons pas en suspens naturellement. Ceux qui pensent pouvoir le faire aisément ne se rendent pas compte qu'ils adoptent bien souvent une position par défaut (default position) qui consiste à lire un texte en tant que fiction lorsque les gages de véracité semblent insuffisants, comme si le lecteur se protégeait instinctivement des manœuvres auctoriales. En effet, et cela va à l'encontre d'un certain nombre d'idées reçues, pour le lecteur, reconstituer le réel d'un autre, comme cela est proposé par l'autobiographie, tenter de saisir ce « là » qui n'est justement plus là, ce passé révolu, voilà où se situe la difficulté ; et c'est l'une des raisons principales qui explique qu'en grande partie, l'autofiction, lorsqu'elle n'est pas polémique (la polémique recentre la lecture sur le réel), est souvent consommée comme de la fiction. Enfin, un narrateur réel qui expose des faits majoritairement fictionnels ne produit pas de l'autofiction : il s'agit soit de modélisation mimétique (si cela est présenté comme tel), et cela relève donc du roman, soit de feintise (non ludique puisque non annoncée), et nous sommes alors dans l'espace familier des autobiographies mensongères. Lorsqu'un texte propose un mélange (modélisation mimétique et récit autobiographique "véridique »), l'effet de contamination exposé plus haut se charge de nous faciliter la tâche. Comme le faisait remarquer Jean-Marie Schaeffer, «le fait que nous soyons dans l'incapacité de distinguer entre réalité et semblant ne change rien au fait que d'un côté il y a la réalité et de l'autre un semblant " (96). Ne pas distinguer revient très souvent à choisir une option et à s'y tenir. Nous sommes en quelque sorte les victimes consentantes de la "tyrannie de la majorité ». Celle-ci détermine la plupart du temps le genre de la globalité du texte. Gérard Genette a explicité avec beaucoup d'à-propos et de clairvoyance la nature de cette règle lorsqu'on l'applique à la fiction :

La fiction n'est guère que du réel fictionnalisé, et la définition de son discours en termes illocutoires ne peut être que fluctuante, ou globale et synthétique: ses assertions ne sont clairement pas toutes feintes, et aucune d'elles peut-être ne l'est rigoureusement et intégralement [...]. Il en est sans doute de même de la fiction comme discours que de la fiction comme entité, ou comme image : le tout y est plus fictif que chacune de ses parties ${ }^{45}$.

18 Il en est de même pour l'autobiographie : celle-ci aura beau être occasionnellement mensongère, elle ne changera pas pour autant d'identité générique.

En conclusion, le panfictionalisme sous toutes ses formes théoriques a sous-estimé un certain nombre de réalités cognitives dont il faut rappeler brièvement ici la nature :

20 - La flexibilité herméneutique du lecteur « moyen » ne le destine pas à maintenir une capacité négative pendant toute la lecture d'un texte.

- Notre tendance naturelle est à la crédulité, la lecture d'une autobiographie comme 
reconstitution d'un « quoi » et d'un « où » bien réels exige de notre part un effort que la fiction ne nécessite pas forcément, lorsque sa mimésis est aisément accessible.

- Dans un contexte référentiel "alternatif», le tout, ou plus exactement ce que le lecteur perçoit comme la majorité générique d'un texte, déterminera l'identité de celuici, et même de ses parties hétérogènes.

21 Distinguer le vrai du faux est une aptitude que nous avons acquise lors de notre petite enfance et qui, à travers le réflexe cognitif d'identification des sources, nous pousse à identifier notre interlocuteur et à en déterminer les intentions. L'existence d'un moi autofictionnel, qui est à la fois réel et fictionnel, relève d'une réalité qui est forcément « autre ». Et si, comme dans une majorité de cas, l'identité de l'autofictionniste est bien réelle, mais les faits rapportés ne le sont que de manière alternative et minoritaire, il est probable qu'il sera avant tout perçu comme un romancier (à tendance autobiographique).

Ceci nous mène à notre dernier point: le tout est déterminé par la base énonciative, c'est-à-dire l'identité du narrateur telle qu'elle est perçue par le lecteur. La fonction auteur demeure déterminante dans l'application de la règle de la majorité et donc dans l'identité générique du texte. Jean-Marie Schaeffer trouve chez Aristote une analyse toute similaire :

23 Même lorsqu'il travaille sur des personnes réelles ou du moins avérées par la tradition, il ne reste pas moins poète mimétique et ne risque pas de passer pour un historien : en effet, il demeure toujours le poiétès d'une structuration mimétique qui est une modélisation généralisante et ne saurait donc être confondue avec un compte-rendu factuel. Aristote va même plus loin, puisqu'il affirme que « même lorsqu'il compose un poème sur des événements réellement arrivés, il n'en est pas moins poète [...] ». (58)

Jean-Marie Schaeffer poursuit, « Aristote a une confiance absolue dans l'immunisation réciproque du monde de la fiction et du monde de la réalité historique » (58). Cette immunisation n'est bien entendu jamais infaillible, mais le philosophe grec avait peutêtre pressenti certaines dispositions cognitives chez le lecteur amenant ce dernier à prendre la plus grande partie pour le tout du fait d'une plasticité cérébrale tout de même limitée. En effet, selon Armstrong, la réponse de notre cerveau à l'hybridité indéterminée serait de la découper en séquences alternatives, et donc d'en nier l'existence : «se raccrocher à une seule lecture à la fois tout en maintenant la capacité de changer de cadres disponible ». Bref, toujours selon Armstrong, « le cerveau ne peut pas maintenir son attention sur deux configurations qui s'excluent mutuellement » et doit «faire volte-face ${ }^{46} »$ constamment. Et si « la capacité du cerveau d'alterner entre des lectures contradictoires est un reflet de son mode de fonctionnement normal ${ }^{47}$ », la contamination peut alors être perçue comme un relâchement, notre réponse à la fatigue cognitive que peut entraîner une alternance irrésolue. 


\section{NOTES}

1. Je me permets de renvoyer notamment à mon ouvrage : Arnaud Schmitt, Je Fictif / Je Réel : Audelà d'une Confusion Postmoderne, Toulouse, Presses Universitaires du Mirail, 2010.

2. " [...] in the end, ambiguous I-narratives requires us to read referentially or fictionally, and not both at once. I will take a contrary stance : that readers routinely "vacillate" and "oscillate" and even double the speaking voice against the logic of both structure and stricture" (Susan S. Lanser, "The "I" of the Beholder: Equivocal Attachments and the Limits of Structuralist Narratology ", in A Companion to Narrative Theory, J. Phelan \& P.J. Rabinowitz (eds.), London : Blackwell Publishing, 2005, 207).

3. Philippe Lejeune, Signes de vie. Le Pacte autobiographique 2, Paris, Éditions du Seuil, 2005.

4. Linda Hutcheon, A Poetics of Postmodernism, London, Routledge, 1988, 10 : « The most radical boundaries crossed [...] have been those between fiction and non-fiction and - by extension between art and life ".

5. Philippe Gasparini, Est-il Je ? Roman autobiographique et Autofiction, Paris, Éditions du Seuil, 2004 \& Autofiction : Une Aventure du Langage, Paris, Éditions du Seuil, 2008.

6. Je tiens absolument à distinguer l'hybridité comme concept théorique appliqué à des textes problématiques et l'hybridité comme hypothétique nature d'un texte indépendante de toute lecture.

7. Paul John Eakin, Fictions in Autobiography: Studies in the Art of Self-Invention, Princeton, Princeton University Press, 1985, 5: «Fictions and the fiction-making process are a central constituent of the truth of any life as it is lived and of any art devoted to the presentation of that life ».

8. Timothy Dow Adams, Telling Lies in Modern American Autobiography, Chapel hill, The University of North Carolina Press, 1990, 10-11: « The arguments, which stacked fiction against nonfiction, became even more tendentious once the nonfictional novel, the New Journalism, and the postmodern era brought such terms as factoid, pseudofactual, and docudrama ».

9. David Shields, Reality Hunger : A Manifesto, London, Hamish Hamilton, 2010 \& David Shields, How Literature Saved my Life. London, Notting Hill Editions, 2013.

10. «An awful lot of fiction is immensely autobiographical, and a lot of nonfiction is highly imagined " (Reality Hunger, 63).

11. "The line between fact and fiction is fuzzier than most people find it convenient to admit » (Reality Hunger, 65).

12. "Some of the best fiction is now being written as nonfiction » (Reality Hunger, 26).

13. Leigh Gilmore, The Limits of Autobiography: Trauma and Testimony, Ithaca and London, Cornell University Press, 2001, 47 : «Once fiction's truth is preferred to fact's, the authority of both trauma and autobiography that derives from the eyewitness's credibility is thrown into a crisis of legitimacy ».

14. Ronald James Lethcoe in Manfred Jahn, «Frames, preferences, and the reading of thirdperson narratives : towards a cognitive narratology ", Poetics Today, vol. 18, n4, 459: "When reading a passage of continuous discourse the reader tends to adopt the path of least resistance, reading in a such a way to preserve the continuity of the report ».

15. Monika Fludernik, " Narrative and its development in Ulysses », Journal of Narrative Technique, $\mathrm{n}^{\circ} 16,1986,20$ : «Once the reader has established a prevalent perspective, he tends to persevere with it as long as possible».

16. Paul B. Armstrong, How Literature Plays with the Brain: the Neuroscience of Reading and Art, Baltimore, John Hopkins University Press, 2013, 73 : « [...] to routinize repeated operations [...]». 
17. Umberto Eco, Kant et l'Ornithorynque, Paris, Grasset, 1999, 149: «La psychologie cognitive parle souvent de notre pouvoir de penser comme quelque chose de fondé sur la possibilité d'une organisation catégorielle. L'idée est que le monde dont nous avons l'expérience est composé d'une telle quantité d'objets et d'événements que si nous devions les identifier et les nommer tous, nous serions dépassés par la complexité du milieu; ainsi, la seule façon de ne pas devenir « esclave du particulier » tient dans notre capacité de « catégoriser ».

18. «Primacy and recency are cognitive mechanisms that can be profitably explained against the background of frames and preferences. Normally, a frame can be imagined to have two quasiorganic instincts : It tries to protect itself, and it tries to maximize its scope » (Jahn, 457).

19. «It usually takes a long time for neural structures to get established, and a single reading of one particular book is unlikely to transform them » (Armstrong, 139).

20. "Confronted with novelty or anomaly, an interpreter does not erase his or her brain's habitual patterns of response and start over again from scratch but, rather, revises and extends the familiar to accommodate the unfamiliar» (Armstrong, 73).

21. "In neurological terms, each of these three configurations (duck, rabbit, or duck-rabbit) would entail a somewhat different cortical synthesis, creating a different assembly of neurons, each temporarily holding the others at bay [...]» (Armstrong, 68).

22. Daniel Kahneman, Thinking, Fast and Slow, London, Penguin Books, 2011. Pour la traduction française : Système 1/ Système 2 : Les deux vitesses de la pensée, Paris, Flammarion, 2012.

23. «How the brain can experiment depends on the repertoire it already has at its disposal [...] » (Armstrong, 72).

24. Dorrit Cohn, Transparent Minds, Princeton, Princeton University Press, 1978. Pour la traduction française : Dorrit Cohn, La Transparence intérieure, Paris, Éditions du Seuil, 1981.

25. Norman N. Holland, Literature and the Brain, Gainesville (FL), The PsyArt Foundation, 2009.

26. «[...] a special form of object recognition in which the objects are letters, graphemes, margins, words [...] identified as 'out there' in a certain region of space » (32).

27. Jean-Marie Schaeffer, Pourquoi la fiction?, Paris, Éditions du Seuil, 1999, 122.

28. Marie-Laure Ryan, Possible Worlds : Artificial Intelligence, Narrative Theory, Bloomington (IN), The Indiana University Press, 1991, 24.

29. "the sentence of War and Peace "On the twenty-ninth May [1812] Napoleon left Dresden" is verified in AW, but its reference world is the world of War and Peace» (29).

30. « Autobiography might be best thought of as a thing made out of a thing done » (Dow Adams 10).

31. Wilfrid Sellars, Empiricism and the Philosophy of Mind, Cambridge (MA), Harvard University Press, (1956) 1997.

32. Semir Zeki, Inner Vision: An Exploration of Art and the Brain, Oxford, Oxford University Press, 1999,3 : «What we see is determined as much by the organisation and laws of the brain as by the physical reality of the external world ».

33. Antonio Damasio, The Feeling of What Happens : Body and Emotion in the Making of Consciousness, New York, Harcourt Brace, 1999, 127 : «We are probably late for consciousness by about five hundred milliseconds ».

34. Peter Mendelsund, What We See When We Read, New York, Vintage Books, 2014, 415 : «Writers reduce when they write, and readers reduce when they read. The brain itself is built to reduce [...]. Verisimilitude is not only a false idol, but also an unattainable goal ».

35. Paul de Man, “Autobiography as De-facement," Modern Language Notes 94.5 (1979), 919-30.

36. Jean Starobinski, La Relation Critique, Paris, Gallimard, 1970, 92.

37. Damasio, The Feeling of What Happens, 173.

38. Richard J. Gerrig, Experiencing Narrative Worlds: On the Psychological Activities of Reading, New Haven, Yale University Press, 1998. 
39. Brian McHale, Postmodernist Fiction, London and New York, Routledge, 1987, 197 : «Intended to establish an absolute level of reality, it paradoxically relativizes reality ; intended to provide an ontologically stable foothold, it only destabilizes ontology further. For the metafictional gesture of sacrificing an illusory reality to a higher, "realer" reality, that of the author, sets a precedent : why should this gesture not be repeatable? What prevents the author's reality from being treated in its turn as an illusion to be shattered? Nothing whatsoever, and so the supposedly absolute reality of the author becomes just another level of fiction, and the real world retreats to a further remove $"$.

40. Notre mémoire à long terme (long term memory, ou LTM) se scinde elle-même en deux types, mémoire consciente et inconsciente (ou declarative et non-declarative), cette dernière présentant à son tour deux variantes: la mémoire sémantique (semantic memory) qui gère les «informations générales ", « le genre de chose que nous avons apprises à l'école » (Holland, 131), et la mémoire épisodique qui s'appuie sur notre expérience, sur ce que nous avons vécu.

41. Lisa Zunshine, Why We Read Fiction - Theory of Mind and the Novel, Columbus, The Ohio State University Press, 2006.

42. "People have always cared deeply about the difference between "true" and "feign'd" stories and were even willing to die for their right to call a myth a myth» (Zunshine, 69).

43. "Bookstores cling to the separation between "fiction" and, say, "history," in their shelving practices. Can it be that imperfect as it is, this separation saves the customers a significant cognitive effort of "deciding" (subconsciously, of course), when they begin to read a book, how much of metarepresentational tagging each little element of the story will need? (Zunshine, 71).

44. Peter Stockwell, Cognitive Poetics, An Introduction, London, Routledge, 2002.

45. Gérard Genette, Fiction et diction, Paris, Éditions du Seuil, 1991, 60.

46. «But the fact that the brain cannot focus attention on two mutually exclusive configurations simultaneously, but must flip-flop between them, shows that cognition cannot take place by simply opening oneself up to the flux of phenomena » (Armstrong, 74).

47. "The brain's capacity to alternate between conflicting readings is a reflection of its normal functioning [...]» (Armstrong, 77).

\section{ABSTRACTS}

The aim of this article is to reassess the generic ambiguity inherent in autofiction from a cognitive point of view. Indeed, in the wake of the recent debate on autofiction, we would like to examine, not the nature, but the reception of these ambiguous texts. For instance, what is the cognitive basis of panfictionalism, the dominant approach when it comes to hybridity? It is our contention that panfictionalism's dismissal of generic frames remains unrealistic since the notion of frame (narrative schemas, cognitive types, perceptual habits...) is the necessary structure to get to grips with reality (even textual reality). For the "grey zone" to become tangible, the reader must suspend both belief and disbelief, but this indefinite position seems far removed from the reality of reading. What's more, the exponents of hybridity overestimate our cognitive flexibility : there is every reason to believe that quite early in the process of reading a text, we opt for a genre or at least a specific horizon of expectation and, more often than not, stick to it. 
Il s'agit dans cet article de poser la question de l'ambiguïté générique inhérente à l'autofiction d'un point de vue cognitif. En effet, dans le sillage du long débat consacré à la nature même de l'autofiction, il semble maintenant nécessaire d'envisager ces textes tout simplement du point de vue de leur réception. Par exemple, quelle est la réalité cognitive du panfictionalisme, approche dominante en ce qui concerne l'hybridité générique? Nous tenterons de démontrer dans cet article que l'absence de cadre proposée par le panfictionalisme n'est pas de l'ordre du possible, puisque le cadre (schémas narratifs, types cognitifs, habitudes perceptuelles...) est la structure minimale qui nous permet d'appréhender le réel (même textuel). Pour que la "zone grise » devienne une réalité, le lecteur doit adopter une position indéfinie entre crédulité et incrédulité par rapport à ce qu'il lit, mais cette position est souvent très éloignée de la réalité de la lecture. De plus, la notion d'hybridité surestime notre flexibilité herméneutique; tout porte à croire qu'assez tôt dans la lecture d'un texte, nous choisissons une option générique et, la plupart du temps, nous nous y tenons.

\section{INDEX}

Keywords: autofiction, cognitive poetics, hybridity, horizon of expectation, hermeneutical flexibility

Mots-clés: autofiction, poétique cognitive, hybridité, horizon d'attente, flexibilité herméneutique

\section{AUTHOR}

ARNAUD SCHMITT

Université de Bordeaux 